There was no correlation between the occurrence or number of rapid tranquillisation events and patients' general dissatisfaction scores. Side-effects from potent antipsychotic drugs used in rapid tranquillisation incidents did not seem to influence dissatisfaction, although rapid tranquillisation was significantly associated with higher total side-effects over the patient stay. Within those groups who received rapid tranquillisation the polypharmacy group who received a number of different antipsychotics had significantly raised side-effects compared with those receiving monotherapies, either with shortacting haloperidol or intermediate-acting zuclopenthixol acetate alone.

Further research needs to examine the role of ethnic grouping in violence, factors influencing dissatisfaction and remedial and educational aspects of patients' stay on psychiatric intensive care unit. Better prescribing habits avoiding antipsychotic polypharmacy in rapid tranquillisation should also be encouraged.

\section{References}

ATAKAN, Z. (1995) Violence on psychiatric in-patient units: what can be done? Psychiatric Bulletin, 19, 593-596.

HYDE, C. E. \& HARROWER-WILSON, C. (1994) Psychiatric intensive care: principles and problems. Hospital Update, May, 287-295.

PALmSTIERNA, T. \& WistedT, B. (1987) Staff Observation and Aggression Scale, SOAS: Presentation and Evaluation. Acta Psychiatrica Scandinavica, 76, 657-663.

Sheehan, J. D. HARDIE, T. \& WATSON, J. P. (1995) Social deprivation, ethnicity and violent incidents on acute psychiatric wards. Psychiatric Bulletin, 19, 597-599.

Thomas, C. BARTlett, A. \& MezeY, G. C. (1995) The extent and effects of violence among psychiatric in-patients. Psychiatric Bulletin, 19, 600-604.

*Clive E. Hyde, Consultant Psychiatrist, Colina Harrower-Wilson, Sub Unit Nurse Manager and Julie Morris, Senior Medical Statistician, South Manchester University Hospital Trust, Withington Hospital, Nell Lane, West Didsbury, Manchester M20 2LR

*Correspondence

\title{
Benzodiazepine prescribing in a psychiatric hospital
}

\author{
J. Summers and K. W. Brown
}

\begin{abstract}
Using a case note study, this paper presents a longitudinal survey of the effect of psychiatric inpatient care on benzodiazepine prescribing. Standards were proposed to assess the quality of this prescribing. Based on these standards, the study shows inappropriate use of benzodiazepines. Following admission, there was an increase in the number of patients prescribed benzodiazepines and in the number of benzodiazepines prescribed. Of the benzodiazepines withdrawn, most were contrary to the proposed standard. The quality of drug history showed little emphasis being placed on rationalising benzodiazepine prescribing. The issue of how benzodiazepines should be handled during psychiatric admission is discussed.
\end{abstract}

In 1988, the Committee on Safety of Medicines declared that the use of benzodiazepines should be decreased as dependence was becoming a subject of increasing concern (Committee on Safety of Medicines, 1988). Benzodiazepine misuse is a further issue which has raised concern. In 1993, temazepam was the most commonly misused drug in Scotland (Scottish Health Service Common Services Agency, 1993). Furthermore, there is evidence from the Home Office Drugs Inspectorate that "the vast majority of misused benzodiazepines are obtained on prescription" (National Medical Advisory Committee, 1994).

Although anxiolytic benzodiazepine use has decreased to about one-quarter of its use 15 years ago (Royal College of Psychiatrists, 1997). the above concerns remain relevant and emphasise the need for high standards in benzodiazepine prescribing. Psychiatrists should be 
expected to set an example, given the major use of benzodiazepines as psychotropic drugs.

There have been a number of surveys of benzodiazepine prescribing in psychiatric hospitals. However, these have mainly been crosssectional in nature. Muijen \& Silverstone (1987) studied all psychotropics given on one particular day in three different psychiatric hospitals, excluding specialised alcohol wards. The proportion of patients prescribed benzodiazepines ranged from $20 \%$ in a teaching hospital to $52 \%$ in a large mental hospital. These benzodiazepines were usually hypnotics. Other surveys, looking at all psychotropic drugs prescribed on one day and excluding wards dealing with alcohol addiction, gave results within the same range (Michel \& Kolakowska, 1981; Edwards \& Kumar, 1984).

In contrast, there have been few longitudinal studies following the effect of psychiatric inpatient care on benzodiazepine prescribing. Harborne \& Tudor (1995), in an audit of benzodiazepine hypnotic prescribing in one psychiatric hospital, showed $45 \%$ of patients on

Table 1. Diagnoses of the 100 patients studied

\begin{tabular}{ll}
\hline Diagnosis & n \\
\hline Alcohol dependence syndrome & 25 \\
Depressive episode or recurrent & 23 \\
depressive disorder & 18 \\
Paranoid schizophrenia & 9 \\
Bipolar affective disorder & 6 \\
Generalised anxiety disorder & 4 \\
Personality disorder & 3 \\
Paranoid psychosis & 3 \\
Drug dependence syndrome & 2 \\
Schizoaffective disorder & 2 \\
Mixed anxiety and depressive disorder & 2 \\
Alcohol psychosis & 2 \\
Drug psychosis & 1 \\
Dementia of the multi-infarct type & \\
\hline
\end{tabular}

hypnotics at the start of their study. Following implementation of their audit, three subsequent surveys over 18 months showed "a trend towards a decrease in the number of patients taking them on discharge."

\section{Setting a standard}

To address the issue of improving benzodiazepine prescribing, there have been a number of professional guidelines issued. These are mainly based on consensus views and advise that benzodiazepines are only indicated for severe symptoms and, if prescribed, should be at the lowest effective dose and on a short-term basis.

Examples of such guidelines are those of the Committee on Safety of Medicine (1988) and, more recently, those expressed in a report by the National Medical Advisory Committee (1994) on The Management of Anxiety and Insomnia. This report was used in this study to provide a standard to assess the quality of benzodiazepine prescribing. Advice from the report, concerning benzodiazepine prescribing in hospital, included the following.

(a) There should be agreed local guidelines on the prescribing of benzodiazepines, which should conform to the standards published in the British National Formulary (BNF 1993, no. 26).

(b) A full drug history should be taken on admission.

(c) Regular users should not have their medication suddenly withdrawn.

(d) Benzodiazepines should not be prescribed for patients who were not taking them prior to admission unless there are strong and genuine indications.

\section{The study}

The psychiatric records were studied of 100 patients consecutively discharged from the acute

Table 2. Benzodiazepine prescribing following hospital admission

\begin{tabular}{llcl}
\hline & $\begin{array}{l}\text { On admission } \\
n\end{array}$ & $\begin{array}{l}\text { During admission On discharge } \\
n\end{array}$ & $\begin{array}{c}n \\
n\end{array}$ \\
\hline Total number of patients prescribed benzodiazepines & 33 & 68 & 50 \\
Total number of patients prescribed more than one & & & \\
$\quad$ benzodiazepine & 13 & 105 & 21 \\
Total number of benzodiazepines prescribed & 47 & 27 & 71 \\
Temazepam prescriptions & 8 & 28 & 19 \\
Diazepam prescriptions & 11 & 19 & 15 \\
Chlordiazepoxide prescriptions & 7 & 3 & 14 \\
Lorazepam prescriptions & 3 & 14 & 3 \\
Zopiclone prescriptions & 6 & 4 & 10 \\
Buspirone prescriptions & 2 & 13 & 3 \\
Other benzodiazepines and chloral groups & 13 & 10 \\
\hline
\end{tabular}


admission wards of a general psychiatric hospital from 3 January 1994 to 26 February 1994. These included patients treated for alcohol dependence. Information was obtained from general practitioner referral letters, medical and nursing notes, prescription sheets, discharge prescriptions and discharge letters. Benzodiazepine prescribing was recorded on admission, during admission and on discharge. Prescribing of non-benzodiazepine hypnotics and anxiolytics was also included. The standard of note-keeping, concerning benzodiazepine prescribing, was noted on admission.

\section{Findings}

Of the 100 patients, 51 were male. The average age was 49 years (range 17 to 71 years). The diagnoses of the 100 patients are shown in Table 1.

As shown in Table 2, 33\% of patients were prescribed benzodiazepines on admission. This more than doubled to $68 \%$ during admission and remained increased overall at $50 \%$ on discharge.

Table 2 also shows that, of the patients prescribed benzodiazepines, $31 \%$ were prescribed more than one benzodiazepine on admission. This increased to $38 \%$ during admission and increased further to $42 \%$ on discharge. There was a large variety of combinations of benzodiazepines prescribed together totalling 12 . In all cases, the combination consisted of an hypnotic and an anxiolytic.

Table 2 also shows, comparing the type of benzodiazepines prescribed on admission and on discharge, that temazepam prescriptions were more than doubled, diazepam and chlordiazepoxide prescriptions were both increased, and the prescription of the non-benzodiazepine drugs, zopiclone and buspirone, were also increased. Lorazepam prescriptions were unchanged.

Using approximate equivalent daily doses of diazepam (BNF 1993, no. 26), the dosage range of the benzodiazepines prescribed was 2 to $40 \mathrm{mg}$. This did not include the high doses of benzodiazepines used in the more specialised case of alcohol and drug detoxifying regimes.

How the 105 benzodiazepines were prescribed during admission, was compared with the standards provided by the National Medical Advisory Committee (1994) report.

Twenty-six per cent of the benzodiazepines, prescribed during admission, were given as short courses of treatment within BNF guidelines. Most of the benzodiazepines given in this way were prescribed to patients with a diagnosis of alcohol dependence. Nineteen per cent of the benzodiazepines, prescribed during admission, were continued on discharge. These were taken by 17 patients, who had not been taking them prior to admission. Thirty-four per cent of the benzodiazepines, prescribed during admission, had been continued from before admission and were continued after admission at the same dose. Six per cent of benzodiazepine prescriptions were reduced or discontinued during admission within BNF withdrawal guidelines and $15 \%$ were decreased or discontinued during admission contrary to the guidelines.

The standard of drug history was reviewed concerning the 47 benzodiazepines prescribed on admission. The name of the benzodiazepine alone was recorded in $23 \%$ of cases, and the name and the dose of the benzodiazepine in $38 \%$ of cases. In addition to the name and the dose, the indication for treatment was noted in $4 \%$ of cases and the duration of treatment prior to admission was noted in $13 \%$ of cases. There was no record of the patient's view of treatment in any of the notes.

\section{Comment}

This study shows over two-thirds of patients being prescribed benzodiazepines during admission to acute psychiatric wards. Once patients with an admission diagnosis of alcohol dependence are excluded, the proportion is similar to that found in the large mental hospital surveyed by Muijen \& Silverstone (1987).

The longitudinal nature of this study provided additional information about benzodiazepine prescribing and showed practice which failed to conform with consensus standards. In particular, $17 \%$ of the patients were discharged on benzodiazepines newly started in hospital, temazepam prescriptions were more than doubled following admission and, of the benzodiazepines withdrawn, most were contrary to the proposed standards.

However, the issue of benzodiazepine prescribing to psychiatric in-patients may be more complex than this. The standards in this paper advise about the use of benzodiazepines in the management of severe anxiety and/or insomnia (National Medical Advisory Committee, 1994). As these symptoms commonly complicate mental disorder, an increase in benzodiazepine prescribing during psychiatric admission may be appropriate in some cases.

Furthermore, the advice does not include the more specialised uses of benzodiazepines in psychiatry. Examples are in the management of alcohol or drug withdrawal and the use of lorazepam in the treatment of acute agitation in patients with schizophrenia (although, in this paper, no lorazepam was used in this way).

There are also more debatable indications, such as in the use of clonazepam in the treatment 
of tardive dyskinesia (Thaker et al, 1990) and in the use of anxiolytics in the treatment of chronic severe anxiety where, in a small number of cases, "long term treatment may offer the best therapeutic option" (Humphreys \& Hallström, 1995). The Royal College of Psychiatrists (1997) echoes this advice concerning "chronic treatment-resistant anxiety". It even goes further and suggests a similar "pragmatic approach" to chronic hypnotic use. It also states that, in rare cases, "long-term prescriptions of benzodiazepines may be seen as maintenance treatment or harm-limitation in patients who would otherwise consume illicit benzodiazepines".

The issue of withdrawing benzodiazepines during psychiatric hospital admission can also be complicated. Following chronic use, this can be a long-term process. Therefore, it is unlikely to be possible during a hospital admission. However, admission may provide the opportunity to initiate the process.

Ultimately, how practice appears relies on the quality of record-keeping. This is especially the case for the more debatable uses of benzodiazepines as above. Certain practice may be clinically justified but may appear inappropriate if not supported by adequate clinical notes. The importance of putting benzodiazepine prescribing in 'context' has also been highlighted in general practice (Buetow et al, 1996).

There is also a particular need in benzodiazepine prescribing for good communication between hospital doctor, patient and general practitioner. The Royal College of Psychiatrists (1997) emphasises the importance of evaluating the relative benefits and risks of continued prescription in each individual case early in treatment in conjunction with the patient. This highlights how necessary good communication is on admission, but it is also the case on discharge. In the present study, five of the 16 benzodiazepines, withdrawn contrary to guidelines, were done so on discharge and there was no indication of the general practitioner being informed of this.

If a patient is discharged on a benzodiazepine, the discharge letter should state the indication for treatment and its intended duration. In the case of one of the more debatable indications, it may be best to discuss this verbally with the general practitioner. It would also be important to record the patient's view of this.

Good record-keeping and communication is all the more important in benzodiazepine prescribing because of the additional issues of dependence and abuse.

In conclusion, admission to hospital may provide an opportunity to rationalise benzodiazepine prescribing in collaboration with patient and general practitioner. This should be based on locally agreed guidelines, as promoted by the National Medical Advisory Committee (1994), and further practice should be subjected to audit. Such guidelines should accommodate the more specialised uses of benzodiazepines in psychiatry. They should also emphasise the particular importance of record-keeping and communication concerning benzodiazepine prescribing.

In the hospital where the study was based, there were no local guidelines concerning benzodiazepine prescribing. This was despite statistics from three months in 1993 showing that, in the hospital's local health board, more benzodiazepines were prescribed per 1000 patients than in any other Scottish health board (National Medical Advisory Committee, 1994).

Clearly these findings require replication to ascertain whether the inappropriate use of benzodiazepines is a widespread problem.

\section{References}

Buetow, S. A., Sibbald, B., Cantrill, J. A., et al (1996) Prevalence of potentially inappropriate long term prescribing in general practice in the United Kingdom 1980-1995: systematic literature review. British Medical Journal, 313, 1371-1374.

COMMITTEE ON SAFETY OF MEDICINES (1988) Benzodiazepines. Dependence and Withdrawal Symptoms. Current problems, no. 21. London: Medicines Control Agency.

EDWARDS, S. \& KUMAR, V. (1984) A survey of prescribing psychotropic drugs in a Birmingham psychiatric hospital. British Journal of Psychiatry, 145, 502-507.

HARBORNE, G. \& TUDOR, L. (1995) How to change prescribing of hypnotics. Psychiatric Bulletin, 19, 155-157.

HuMPHREYS, S. \& HALLTRÓM, C. (1995) Benzodiazepine dependence and withdrawal - an update. Primary Care Psychiatry, 1, 99-105.

MiCHEL, K. \& KALAKOWSKA. T. (1981) A survey of prescribing psychotropic drugs in two psychiatric hospitals. British Journal of Psychiatry, 138, 217-221.

MulJEN, M. \& SIlverstone, T. A. (1987) Comparative hospital survey of psychotropic drug prescribing. British Journal of Psychiatry. 150, 501-504.

NATIONAL MEDICAL ADVISORY COMMTTTE (1994) The Management of Anxiety and Insomnia. A Report by the National Medical Advisory Committee. Edinburgh: HMSO.

ROYAL COLLEGE OF PSYCHIATRISTS (1997) Benzodiazepines: Risks, Benefits or Dependence. A Re-Evaluation (Council Report CR59). London: Royal College of Psychiatrists.

Scottish Health Service Common Services Agency, INFORMATION AND STATISTICS DIVISION (1993) Scottish Drug Misuse Bulletin. Section 2.5. Edinburgh: ISD Publications.

Thaker, G. K., Nguyen, J. A., Strauss, M. E., et al (1990) Clonazepam treatment of tardive dyskinesia: A practical GABAmimetic strategy. American Journal of Psychiatry. 147, 445-451.

*John Summers, Senior Registrar in General Psychiatry, Larkfield Centre, Garngaber Avenue, Lenzie G66 3UG and Keith W. Brown. Consultant in General Psychiatry and Research Adviser. Central Scotland Healthcare NHS Trust, Larbert

*Correspondence 